\title{
Probing Protein Dynamics Using Multifield Variable Temperature NMR Relaxation and Molecular Dynamics Simulation
}

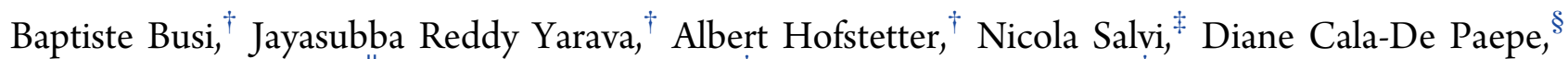

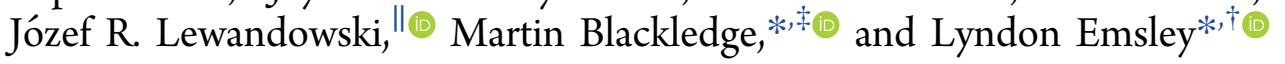 \\ ${ }^{\dagger}$ Institut des Sciences et Ingénierie Chimiques, Ecole Polytechnique Fédérale de Lausanne (EPFL), 1015 Lausanne, Switzerland \\ ${ }^{\ddagger}$ Université Grenoble Alpes, CNRS, CEA, IBS, 38000 Grenoble, France \\ ${ }^{\S}$ Université de Lyon, Institut des Sciences Analytiques (UMR 5280 CNRS/UCBL/ENS Lyon), Centre de RMN à Très Hauts \\ Champs, 69199 Villeurbanne, France \\ "Department of Chemistry, University of Warwick, Coventry CV4 7AL, U.K.
}

\section{Supporting Information}

ABSTRACT: Understanding the interplay between protein function and dynamics is currently one of the fundamental challenges of physical biology. Recently, a method using variable temperature solid-state nuclear magnetic resonance relaxation measurements has been proposed for the simultaneous measurement of 12 different activation energies reporting on distinct dynamic modes in the protein GB1. Here, we extend this approach to measure relaxation at multiple magnetic field strengths, allowing us to better constrain the motional models and to simultaneously evaluate the robustness and physical basis of the method. The data reveal backbone and side-chain motions, exhibiting low- and

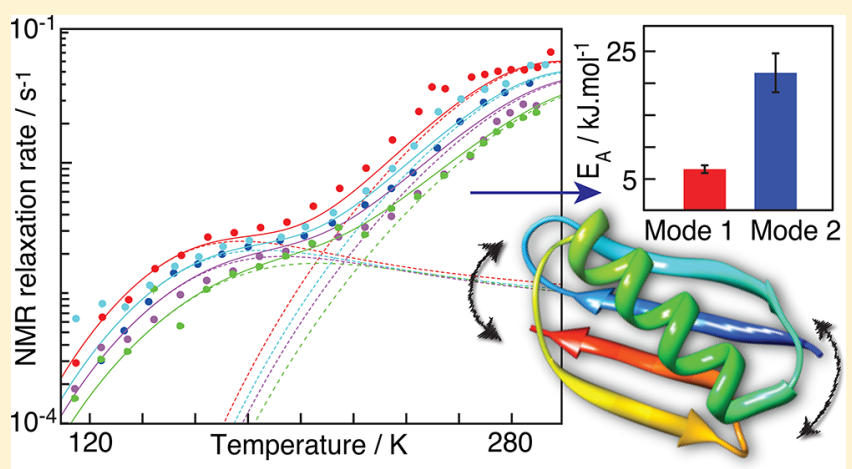
high-energy modes with temperature coefficients around 5 and $25 \mathrm{~kJ} \cdot \mathrm{mol}^{-1}$. The results are compared to variable temperature molecular dynamics simulation of the crystal lattice, providing further support for the interpretation of the experimental data in terms of molecular motion.

\section{INTRODUCTION}

Proteins play a crucial role in almost all biological processes. Knowledge of the protein structure and dynamics is required to understand these processes at the molecular level. ${ }^{1}$ Protein motions at physiological temperature are governed by many distinct dynamic modes occurring at different time scales ranging from picoseconds to seconds. Directly probing these different motional modes and their possible intercorrelation can provide essential insight into the nature of the intrinsic free energy landscape governing the dynamic nature of the protein and its surrounding solvent. ${ }^{2}$

Protein dynamics have been the subject of intense research. $^{2-4}$ The hierarchy of protein dynamics is difficult to access and has been studied using a wide range of pioneering techniques, such as differential scanning calorimetry, ${ }^{5,6}$ terahertz spectroscopy, ${ }^{7,8}$ Mössbauer spectroscopy, ${ }^{9,10}$ dielectric spectroscopy, ${ }^{5,9}$ fluorescence resonance energy transfers, ${ }^{11}$ $\mathrm{X}$-ray crystallography, ${ }^{12-14}$ neutron scattering, ${ }^{15-17}$ and nuclear magnetic resonance (NMR). ${ }^{18}$ Temperature-dependent studies have detected so-called dynamical transitions, between distinct regimes, reporting on the appearance of dominant dynamic modes, possibly related to function, at temperatures ranging from 180 to $240 \mathrm{~K}^{5,8,19-23}$
Because of its sensitivity to a wide range of motional time scales and its site selectivity, NMR can provide considerable insight into these phenomena, allowing the development of a complete and coherent model of protein dynamics over a wide range of temperatures. Liquid state NMR already provides very detailed information about molecular motion around physiological temperatures. ${ }^{24,25}$ However, the investigation of dynamics over a large temperature range is only accessible to solid-state NMR of biological systems, particularly in the case of cryo-temperatures.

Recently, solid-state NMR methods have been developed to an extent which enables the study of site-specific ${ }^{13} \mathrm{C},{ }^{15} \mathrm{~N}$, and ${ }^{1} \mathrm{H}$ relaxation rates in proteins. ${ }^{26}$ Notably, Lewandowski et al. ${ }^{18}$ presented a solid-state NMR approach to study the hierarchy of protein motions. They measured, in a single sample of the protein GB1, a set of 13 different NMR relaxation observables, sensitive to motions occurring on fast and slow time scales and in different parts of the system, over a temperature range from 105 to $280 \mathrm{~K}^{18}$ The data obtained were analyzed by fitting the measured ${ }^{1} \mathrm{H},{ }^{15} \mathrm{~N}$, and ${ }^{13} \mathrm{C}$ relaxation rates to a sum of spectral

Received: September 3, 2018

Revised: September 28, 2018

Published: October 2, 2018 
density functions related to distinct Arrhenius-dependent terms, describing the superposition of distinct motional modes with different locations, timescales, and activation energies. This study provided unique insight into the temperature-dependent activation of distinct dynamic processes within the protein crystal.

This initial investigation measured longitudinal and transverse spin relaxation at a single magnetic field strength, limiting our ability to unambiguously identify the nature of the observed relaxation phenomena and to cross-validate our interpretation in terms of dynamic behavior. Here, again using the protein GB1, we therefore extend the approach to measure longitudinal relaxation over a range of different magnetic field strengths and temperatures, thereby exploiting the dependence of relaxation rates on the Larmor frequency expected from the Redfield theory. This allows us to test the dynamic origin of the measurements, to better constrain the motional models used to interpret the data, and to evaluate the reproducibility of the method. Specifically, we perform the same set of experiments at four different magnetic field strengths (9.4, $11.75,14.1$ and $18.8 \mathrm{~T}$ ), on different sample batches and for different temperature variation protocols. The field-dependent measurements allow a more precise description of the timescale and amplitudes of the backbone motions in GB1, substantiating and refining our findings from the previous single-field analysis.

To further investigate the physical origin of the observations, we performed molecular dynamics (MD) simulations of the crystalline lattice at four temperatures from 248 to $278 \mathrm{~K}$. The results demonstrate almost a negligible change in the amplitude of relaxation-active backbone motions and suggest that the steep change in longitudinal relaxation rates within this range is indeed because of significant slowing of intermediate timescale (nanosecond) backbone motions with decreasing temperature.

\section{EXPERIMENTAL SECTION}

Measurement of Relaxation Rates. An extensive description of the relaxation measurements and the equations used for interpretation are included in the Supporting Information.

Reproducibility. The robustness of the reproducibility experiments presented in Figure 1 was also assessed through cross-validation. The model was fit to $90 \%$ of the $R_{1}$ values (training set), randomly sampled from the three combined sets and used to predict the remaining $10 \%$ (validation set). The full procedure is detailed in the Supporting Information, and Figure $S 6$ shows the experimental against predicted $R_{1}$ values. The root-mean-square deviation between experimental and predicted $R_{1}$ is around $10 \%$ for both training and validation sets, demonstrating that the three sets are interchangeable, and the method is indeed reproducible.

MD Simulation. Three independent $200 \mathrm{~ns}$ trajectories of a periodic box containing 32 explicit copies of the protein comprising eight asymmetric units of four proteins (details in the Supporting Information), ${ }^{35}$ were run at four temperatures from 248 to $278 \mathrm{~K}$. Angular correlation functions describing the motion of internuclear backbone $\mathrm{NH}$ bond vectors were modeled by fitting to a three-exponential fit (see the Supporting Information), in order to extract motional amplitudes and characteristic timescales. The full procedure of the MD simulations is included in the Supporting Information.

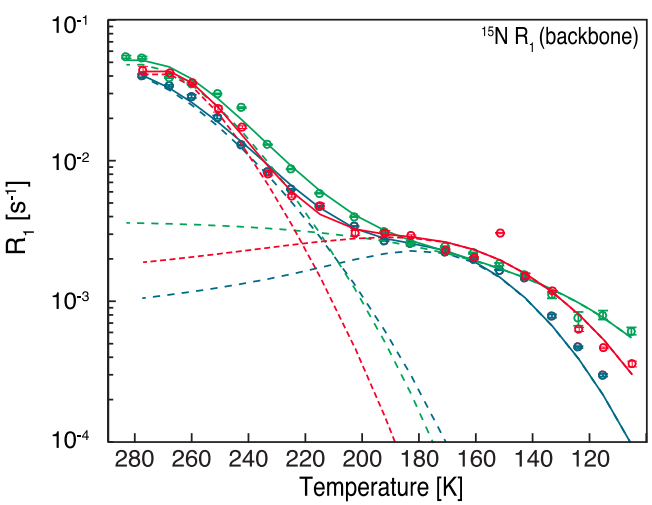

Figure 1. Three sets of measured ${ }^{15} \mathrm{~N} R_{1}$ rates obtained at $11.7 \mathrm{~T}$ as a function of temperature. The error bars reflect the uncertainty in the fit to the data as described in the Experimental Section. Red circles show data set $I^{18}$ the blue circles data set II, and the green circles show data set III. Dashed lines show the contribution due to two motional modes, and the sum is shown as the solid line, where data were fit to a two-component relaxation model. The number of components was statistically justified with an F-test $(p<0.05)$.

Other Experimental Details. Other computational details, experimental data, and the model description are also included in the Supporting Information.

\section{RESULTS AND DISCUSSION}

We initially verified that published data were reproducible. Figure 1 shows ${ }^{15} \mathrm{~N} R_{1}$ measured a function of temperature in three different experiments. The red symbols correspond to the data from Lewandowski et al. ${ }^{18}$ recorded in Lyon in 2012 (here called set I), and the blue symbols are the $R_{1}$ rates extracted from data recorded in Lausanne with a different sample of GB1 and progressively increasing temperatures (here called set II). Green symbols show rates from experiments on the same sample, performed two months later on the same spectrometer using a random order of temperatures (here called set III). The same temperature behavior is observed in all three datasets that can all be fit to two motional modes, one with low activation energy dominant at low temperatures and one with high activation energy appearing at higher temperatures (Figure S4). The activation energies and correlation times match well between the three samples, and there is no evidence for hysteresis in the temperature dependence. Similar results are found for activation energies and correlation times extracted for the other five relaxation rates (Figure S3 and Table S1). Note that while the energy barriers are accurately defined, the motional timescales are less accurate. ${ }^{27}$ As described in the Methods section above, the robustness of the reproducibility experiments was confirmed through crossvalidation.

The magnetic field dependence of the temperature-dependent relaxation measurements was also investigated. The pronounced Larmor frequency dependence of longitudinal relaxation, particularly in the nanosecond timescale range, (see the Supporting Information) suggests that measuring data at multiple fields should provide a significantly more accurate analysis, allowing us to test the interpretation of the observed relaxation phenomena in terms of dynamic reorientation of relaxation active interactions. This corresponds to a more accurate mapping of the spectral density function, or in more poetic terms, making sure that the light shines on all the motional modes. ${ }^{28,29}$ 
Figure 2 shows relaxation rates from ${ }^{15} \mathrm{~N}$ lysine sidechain moieties, indicating that relaxation shows similar trends as a

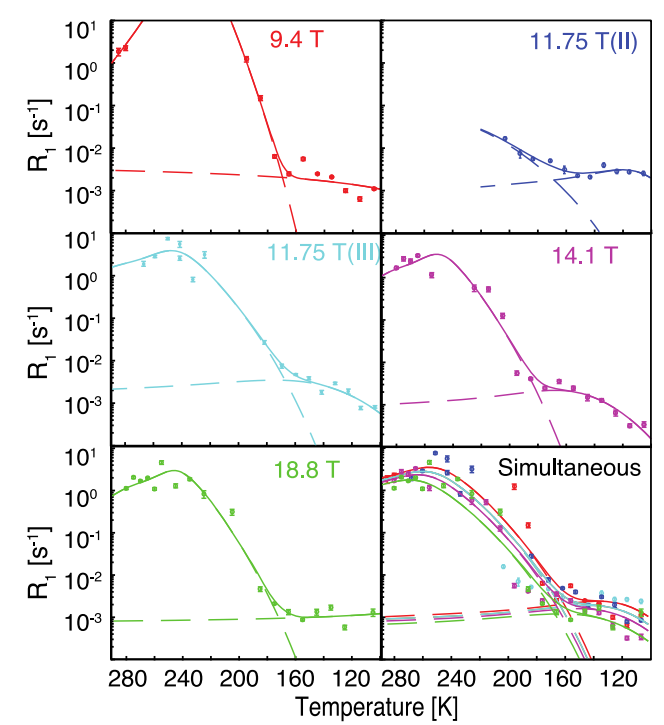

Figure 2. ${ }^{15} \mathrm{~N}_{\mathrm{Lys}} R_{1}$ rates measured as function of temperature and magnetic field strength. Data were recorded from 105 to $285 \mathrm{~K}$, and at 9.4 T, $11.75 \mathrm{~T}$ (set II), $11.75 \mathrm{~T}$ (set III), $14.1 \mathrm{~T}$ and $18.8 \mathrm{~T}$. The error bars reflect the error on the $R_{1}$ fits. Dashed lines show contributions of a given motional mode to relaxation, and the contribution from the sum of the modes is shown as the solid line, which is fit to the relaxation data (points). The number of modes needed (here 2) for the fits was statistically justified with an F-test $(p<0.05)$, except for the $11.75 \mathrm{~T}$ set III data, where two modes were also used for comparison purposes. The first five panels show the temperature dependent relaxation fit to the relaxation rates obtained at a single magnetic field. The sixth panel shows the temperature-dependent relaxation fit simultaneously to all magnetic fields.

function of temperature at all the different fields. Analysis of all data in terms of two contributions to the spectral density function reveal clear clustering, both in the correlation times and activation energies of the two extracted motional modes (Figure S5). For ${ }^{15} \mathrm{~N}_{\text {Lys }}$ solvent rotations become active at around $160 \mathrm{~K}$, which allows side-chains to access different rotameric states on time scales, which interfere with the pulse sequence and leads to the disappearance of the ${ }^{15} \mathrm{~N}_{\text {Lys }}$ resonance signals in the region between 160 and $240 \mathrm{~K}^{30,31}$ This results in large errors and significant differences in the values extracted from the fits to the motional modes at each field. By combining the information obtained at different fields and fitting them simultaneously, we are able to determine the motional parameters with much greater accuracy.

Figure 3 summarizes the field-dependent variable-temperature data for all six measured relaxation rates (individual curves and rates given in the Supporting Information). ${ }^{15} \mathrm{~N} R_{1}$ rates, which report on motion in the protein backbone, ${ }^{32}$ or the ${ }^{13} \mathrm{C}$ methyl $R_{1}$ measurements, reporting on hydrophobic side chains, show very clear field dependencies that are well reproduced in the joint fits. The figure also shows the modes that are found as a result of the joint fits to the data. Again, using the information from all fields simultaneously leads to a much more precise determination of the motional parameters from all the measured rates. For example, the $R_{1}$ maximum is better defined at $9.4 \mathrm{~T}$, leading to a more precise estimate of both timescales and amplitudes, as is evident in Figure 3. Both the decreasing $R_{1}$ and the observed shift of $R_{1}$ maximum with

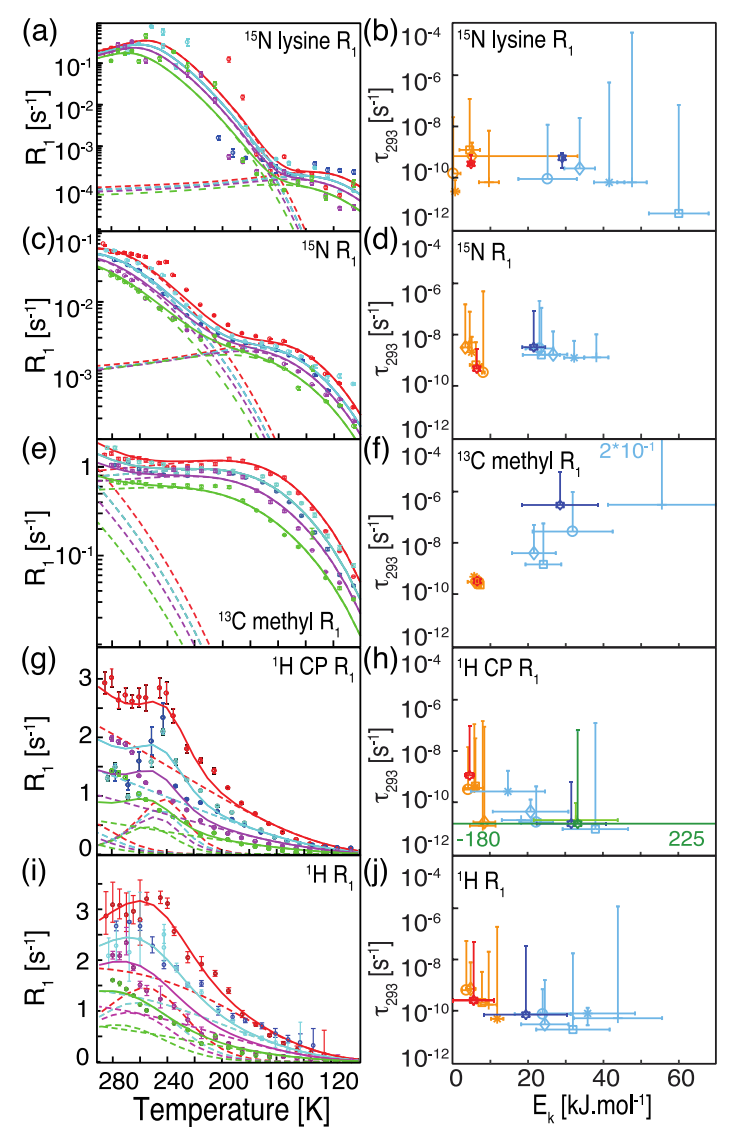

Figure 3. Left panels show the longitudinal relaxation rates as function of temperature for all investigated nuclei. Data were recorded from 105 to $285 \mathrm{~K}$. The error bars reflect the error on the $R_{1}$ fits. Dashed lines show contributions of a given motional mode to relaxation, and the contribution from the sum of the modes is shown as the solid line, which is fit to the relaxation data (points) for all fields simultaneously. The color code is given as: red $9.4 \mathrm{~T}$, dark blue 11.75 $\mathrm{T}$ (set II), cyan $11.75 \mathrm{~T}$ (set III), purple $14.1 \mathrm{~T}$, and green $18.8 \mathrm{~T}$. The number of modes was statistically justified with an F-test $(p<$ 0.05 ). The right panels show the activation energy plotted against the motional rates $\tau_{293}$ for all the investigated relaxation rates. Dark symbols (blue, red, and green) show the simultaneous fit over all magnetic fields, whereas the light symbols (cyan, orange, and green) show the fits at a single magnetic field. Error bars are set to \pm 1 standard deviation, calculated with a Monte Carlo estimation over 500 runs. In the right panels the symbols represent: $9.4 \mathrm{~T}$ (square), 11.75 T (set II) (diamond), 11.75 T (set III) (circle), 14.1 T (cross), and 18.8 $\mathrm{T}$ (asterisk) and denote the values extracted from the single field fits. Bold stars show values extracted from a simultaneous fit to all data. The first modes are represented in orange, the second in blue, and the third in green.

respect to magnetic field strength closely follow theoretically expected behavior, substantiating the dynamic origin of the relaxation phenomenon.

This analysis also illustrates the problems associated with describing the dynamics by a so-called "dynamic transition temperature". ${ }^{15} \mathrm{~N} R_{1}$ and ${ }^{13} \mathrm{C}$ methyl $R_{1}$ relaxation measurements clearly show that the observed "crossover" temperature, where the higher activation energy mode becomes the dominant source of relaxation; it changes as a function of the magnetic field, as expected from theory. This highlights that the apparent "transition temperatures" are properties that depend both on the physical probe and the intrinsic properties of the protein that are better described in terms of the energy 
barriers to the motion. ${ }^{4}$ We note that all but three of the curves can be explained with two motional modes. We also find that ${ }^{13} \mathrm{C}^{\prime} R_{1}$ only required a single mode to explain the data, as discussed below.

From Figure 4 we note that intrinsic ${ }^{13} \mathrm{C}^{\prime} R_{1}$ rates, which would be primarily because of ${ }^{13} \mathrm{C} C S A$, are expected to be

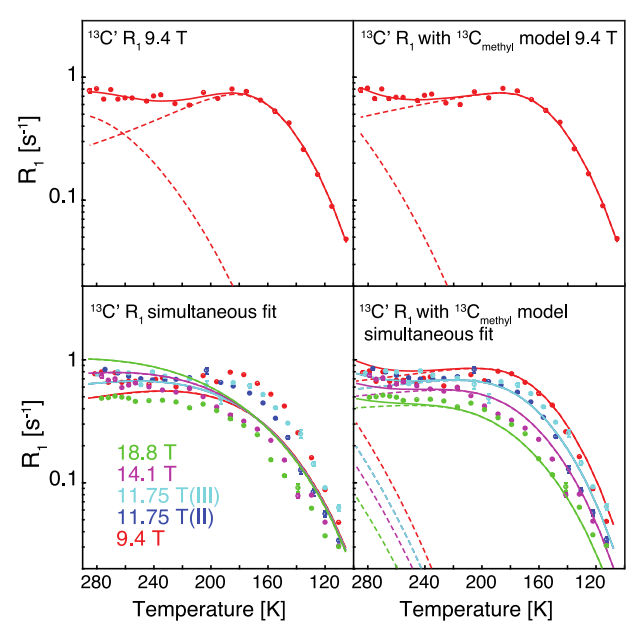

Figure $4 .{ }^{13} \mathrm{C}^{\prime} R_{1}$ rates fitted with two different models: the model for carbonyl relaxation on the left, with the major relaxation contribution coming from CSA, or on the right using the model for ${ }^{13} \mathrm{C}_{\text {methyl }} R_{1}$, with the major contribution to relaxation coming from dipole-dipole interactions. This clearly demonstrates that the major contribution to $R_{1}$ for ${ }^{13} \mathrm{C}^{\prime}$ is due to spin diffusion to nearby fast relaxing methyl groups.

longer than those we observe. Indeed, Lewandowski et al. ${ }^{18}$ had already noted that in a fully carbon-13-enriched and protonated protein, spin diffusion of longitudinal magnetization between nearby carbon atoms is likely to be faster than the $R_{1}$ relaxation rates, and it is well known that this will lead to averaged apparent $R_{1}$ relaxation rates that are dominated by the fastest relaxing local species. ${ }^{33}$ This will be much less significant for transverse relaxation rates or rates between distant or sparse spins such as ${ }^{15} \mathrm{~N} .{ }^{34}$ In the case of ${ }^{13} \mathrm{C}^{\prime} R_{1}$, the apparent rates are likely to be dominated by the faster relaxation of nearby methyl groups. ${ }^{18}$ The multifield data here provide compelling evidence to support this hypothesis. Figure 4 shows that whereas both pure CSA relaxation or dipoledipole relaxation can explain the behavior observed at a single field as a function of temperature, the expected field dependence of the two mechanisms is different and only the dipole-dipole mechanism can correctly explain the field dependence of the ${ }^{13} \mathrm{C}^{\prime} R_{1}$, confirming relayed relaxation of the carbonyls by the methyl sinks. As expected in this analysis, the ${ }^{13} C^{\prime} R_{1}$ are now also explained by two modes with this model, as are the ${ }^{13} \mathrm{C}_{\text {methyl }} R_{1}$.

The ${ }^{1} \mathrm{H}_{\mathrm{CP}} R_{1}$ data justifies the use of three modes. This was also observed in the previous study at a single field and is validated here by the multifield data that fits to the three modes with energy barriers of 5, 34, and $35 \mathrm{~kJ} \mathrm{~mol}^{-1}$. These rates are influenced by both the protein, and the crystal water, which implies that they may indeed be different from, for example, the ${ }^{13} \mathrm{C}_{\text {methyl }} R_{1}$ which report mainly on the hydrophobic sidechain dynamics. ${ }^{18}$

The physical basis of our interpretation of the experimental data was further probed using temperature-dependent $\mathrm{MD}$ simulations of GB1 in the crystalline environment. Motional amplitudes evolve with temperature over a restricted range (Figure 5), where the systematic decrease observed when

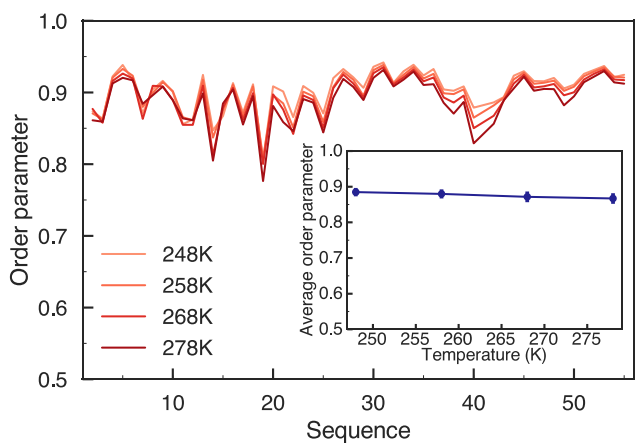

Figure 5. Temperature dependence of dynamic parameters for backbone amide $\mathrm{NH}$ bonds derived from MD simulations. Order parameters: The marginal systematic decrease observed when increasing the temperature is because of the increase of the amplitude of very fast $(<10 \mathrm{ps})$ fluctuations that do not contribute significantly to relaxation (see also Figure S7). Inset: The average order parameter calculated by averaging the values of each of the four curves in the main panel varies only slightly as a function of the temperature (see text).

increasing the temperature is almost entirely because of the increase of the amplitude of very fast $(<10 \mathrm{ps})$ fluctuations that do not contribute significantly to relaxation (see also Figure S8). This indicates that the assumption that motional amplitudes do not change significantly over this temperature range is physically reasonable, supporting the observation derived from experimental data that changes in timescales of relaxation-active motions are principally responsible for the steep change in backbone ${ }^{15} \mathrm{~N} R_{1}$ over the $240-280 \mathrm{~K}$ range. Average characteristic correlation times extracted from the trajectories indeed slow down at lower temperatures (Figure S9), although the timescales are slower than seen experimentally, probably because of limitations in the force field at cryo-temperatures.

Figure 6 compares the two activation energies for the different modes extracted from the multifield analysis (numerical values are given in Table S1). (Note that in the analysis we use here we obtain a single activation energy for each mode per residue type. This description is obviously an approximation, because the relaxation rates measured are almost certainly because of a finite distribution of motions over a range of timescales and amplitudes. The activation energies and amplitudes obtained should thus be considered as weighted averages.) The energies clearly cluster, with a low energy barrier between 5 and $10 \mathrm{~kJ} \mathrm{~mol}^{-1}$ and a high-energy barrier between 20 and $40 \mathrm{~kJ} \mathrm{~mol}^{-1}$. These results are in good agreement with our previous work, ${ }^{18}$ substantiating, in accordance with previous publications, ${ }^{4,9,18}$ the identification of two to three main motional modes per component: a uniform low energy mode $\left(<10 \mathrm{~kJ} \mathrm{~mol}^{-1}\right)$ and one to two high energy $\left(>15 \mathrm{~kJ} \mathrm{~mol}^{-1}\right)$ modes per component. We note that the activation energies of the second mode are not as similar among the different probes as for the first mode. This apparent inhomogeneity can be tentatively assigned to more variability in the high-energy motions as compared to the low-energy motions which appear more homogenous across the protein. Overall these observations are consistent with our previous assignment of the high activation energy components to 


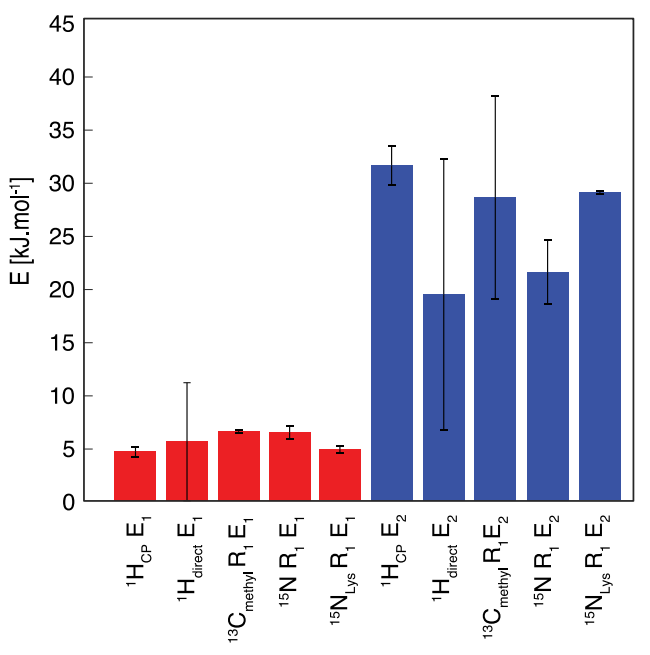

Figure 6. Activation energies from the simultaneous fits, plotted for each nuclear spin probe. The error bars are set to one standard deviation calculated with Monte Carlo error estimation over 500 runs. The red bars represent the energies of the first mode and the blue bars the energies of the second mode. The third mode of the ${ }^{1} \mathrm{H}_{\mathrm{CP}} R_{1}$ is not plotted. The energies for ${ }^{13} \mathrm{C}^{\prime} R_{1}$ are not reported because, as described in the text, spin diffusion renders them equivalent to the ${ }^{13} \mathrm{C}_{\text {methyl }} R_{1}$.

anisotropic motions of peptide planes or side-chain moieties that dominate solution-state spin relaxation in proteins, whereas the low activation energy components appear to report on smaller amplitude librational motions.

\section{CONCLUSIONS}

In summary, we have presented a variable temperature, multifield NMR relaxation approach to disentangle motional modes governing protein dynamics, and used temperaturedependent MD simulation to support the interpretation. The combination of multifield relaxation rates and $\mathrm{MD}$ simulation substantiates the interpretation of ${ }^{15} \mathrm{~N} R_{1}$ relaxation in terms of backbone dynamics and more accurately defines the activation energies and correlation times of the associated motional modes.

\section{ASSOCIATED CONTENT}

\section{(5) Supporting Information}

The Supporting Information is available free of charge on the ACS Publications website at DOI: 10.1021/acs.jpcb.8b08578.

Sample preparation, experimental and computational details, experimental data, relaxation rates and motional models, cross-validation detail, and MD simulation details (PDF)

\section{AUTHOR INFORMATION}

\section{Corresponding Authors}

*E-mail: martin.blackledge@ibs.fr (M.B.).

*E-mail: lyndon.emsley@epfl.ch (L.E.).

\section{ORCID $\odot$}

Józef R. Lewandowski: 0000-0001-6525-7083

Martin Blackledge: 0000-0003-0935-721X

Lyndon Emsley: 0000-0003-1360-2572

Notes

The authors declare no competing financial interest.

\section{ACKNOWLEDGMENTS}

We thank Laura Piveteau, Wei-Chih Liao and Christophe Copéret (ETH Zürich), and Anne Lesage and David Gajan (CRMN Lyon) for the access to and help regarding their spectrometers. This work was supported by the Swiss National Science Foundation grant number 200021_162514 and Advanced Postdoc Mobility Fellowship P300P2_167742 (NS), and ERC-2015-CoG 648974. MD simulations were performed using the HPC resources of CCRT available by GENCI (Grand Equipement National de Calcul Intensif, project number A0030707486).

\section{REFERENCES}

(1) Anderson, A. C. The process of structure-based drug design. Chem. Biol. 2003, 10, 787-797.

(2) Khodadadi, S.; Sokolov, A. P. Atomistic details of protein dynamics and the role of hydration water. Biochim. Biophys. Acta 2017, 1861, 3546-3552.

(3) Buck, M.; Boyd, J.; Redfield, C.; MacKenzie, D. A.; Jeenes, D. J.; Archer, D. B.; Dobson, C. M. Structural determinants of protein dynamics: Analysis of $15 \mathrm{~N}$ NMR relaxation measurements for mainchain and side-chain nuclei of hen egg white lysozyme. Biochemistry 1995, 34, 4041-4055.

(4) Fenimore, P. W.; Frauenfelder, H.; Magazù, S.; McMahon, B. H.; Mezei, F.; Migliardo, F.; Young, R. D.; Stroe, I. Concepts and problems in protein dynamics. Chem. Phys. 2013, 424, 2-6.

(5) Jansson, H.; Swenson, J. The protein glass transition as measured by dielectric spectroscopy and differential scanning calorimetry. Biochim. Biophys. Acta 2010, 1804, 20-26.

(6) Jansson, H.; Bergman, R.; Swenson, J. Role of solvent for the dynamics and the glass transition of proteins. J. Phys. Chem. B 2011, 115, 4099-4109.

(7) Lipps, F.; Levy, S.; Markelz, A. G. Hydration and temperature interdependence of protein picosecond dynamics. Phys. Chem. Chem. Phys. 2012, 14, 6375-6381.

(8) Knab, J. R.; Chen, J.-Y.; He, Y.; Markelz, A. G. Terahertz measurements of protein relaxational dynamics. Proc. IEEE 2007, 95, $1605-1610$.

(9) Frauenfelder, H.; Chen, G.; Berendzen, J.; Fenimore, P. W.; Jansson, H.; McMahon, B. H.; Stroe, I. R.; Swenson, J.; Young, R. D. A unified model of protein dynamics. Proc. Natl. Acad. Sci. U.S.A. 2009, 106, 5129-5134.

(10) Parak, F.; Formanek, H. Untersuchung des schwingungsanteils und des kristallgitterfehleranteils des temperaturfaktors in myoglobin durch vergleich von mössbauer-absorptionsmessungen mit röntgenstrukturdaten. Acta Crystallogr., Sect. A: Found. Adv. 1971, 27, 573578.

(11) Roy, R.; Hohng, S.; Ha, T. A practical guide to single-molecule FRET. Nat. Methods 2008, 5, 507-516.

(12) Rasmussen, B. F.; Stock, A. M.; Ringe, D.; Petsko, G. A. Crystalline ribonuclease A loses function below the dynamical transition at 220 K. Nature 1992, 357, 423-424.

(13) Weik, M.; Colletier, J.-P. Temperature-dependent macromolecular X-ray crystallography. Acta Crystallogr., Sect. D: Biol. Crystallogr. 2010, 66, 437-446.

(14) Tilton, R. F.; Dewan, J. C.; Petsko, G. A. Effects of temperature on protein structure and dynamics: $\mathrm{x}$-ray crystallographic studies of the protein ribonuclease-A at nine different temperatures from 98 to 320K. Biochemistry 2002, 31, 2469-2481.

(15) Gaspar, A. M.; Appavou, M.-S.; Busch, S.; Unruh, T.; Doster, W. Dynamics of well-folded and natively disordered proteins in solution: a time-of-flight neutron scattering study. Eur. Biophys. J. 2008, 37, 573-582.

(16) Doster, W.; Bachleitner, A.; Dunau, R.; Hiebl, M.; Lüscher, E. Thermal-properties of water in myoglobin crystals and solutions at subzero temperatures. Biophys. J. 1986, 50, 213-219. 
(17) Doster, W.; Cusack, S.; Petry, W. Dynamical transition of myoglobin revealed by inelastic neutron scattering. Nature 1989, 337, 754-756.

(18) Lewandowski, J. R.; Halse, M. E.; Blackledge, M.; Emsley, L. Direct observation of hierarchical protein dynamics. Science 2015, $348,578-581$.

(19) Doster, W. The dynamical transition of proteins, concepts and misconceptions. Eur. Biophys. J. 2008, 37, 591-602.

(20) Frauenfelder, H.; Young, R. D.; Fenimore, P. W. Dynamics and the free-energy landscape of proteins, explored with the Mossbauer effect and quasi-elastic neutron scattering. J. Phys. Chem. B 2013, 117, 13301-13307.

(21) Idiyatullin, D.; Nesmelova, I.; Daragan, V. A.; Mayo, K. H. Heat capacities and a snapshot of the energy landscape in protein GB1 from the pre-denaturation temperature dependence of backbone $\mathrm{NH}$ nanosecond fluctuations. J. Mol. Biol. 2003, 325, 149-162.

(22) Teeter, M. M.; Yamano, A.; Stec, B.; Mohanty, U. On the nature of a glassy state of matter in a hydrated protein: Relation to protein function. Proc. Natl. Acad. Sci. U.S.A. 2001, 98, 11242-11247.

(23) Vugmeyster, L.; Ostrovsky, D.; Ford, J. J.; Lipton, A. S. Freezing of dynamics of a methyl group in a protein hydrophobic core at cryogenic temperatures by deuteron NMR spectroscopy. J. Am. Chem. Soc. 2010, 132, 4038-4039.

(24) Mittermaier, A.; Kay, L. E. New tools provide new insights in NMR studies of protein dynamics. Science 2006, 312, 224-228.

(25) Abyzov, A.; Salvi, N.; Schneider, R.; Maurin, D.; Ruigrok, R. W. H.; Jensen, M. R.; Blackledge, M. Identification of dynamic modes in an intrinsically disordered protein using temperature-dependent NMR relaxation. J. Am. Chem. Soc. 2016, 138, 6240-6251.

(26) Mack, J. W.; Usha, M. G.; Long, J.; Griffin, R. G.; Wittebort, R. $\mathrm{J}$. Backbone motions in a crystalline protein from field-dependent $2 \mathrm{H}$ NMR relaxation and line-shape analysis. Biopolymers 2000, 53, 9-18.

(27) Beckmann, P. A.; Buser, C. A.; Mallory, C. W.; Mallory, F. B.; Mosher, J. Methyl reorientation in solid 3-ethylchrysene and 3isopropylchrysene. Solid State Nucl. Magn. Reson. 1998, 12, 251-256.

(28) Smith, A. A.; Ernst, M.; Meier, B. H. Because the light is better here: Correlation-time analysis by NMR spectroscopy. Angew. Chem., Int. Ed. 2017, 56, 13590-13595.

(29) Peng, J. W.; Wagner, G. Mapping of the spectral densities of N$\mathrm{H}$ bond motions in eglin- $\mathrm{C}$ using heteronuclear relaxation experiments. Biochemistry 1992, 31, 8571-8586.

(30) Lee, A. L.; Wand, A. J. Microscopic origins of entropy, heat capacity and the glass transition in proteins. Nature 2001, 411, 501504.

(31) Ni, Q. Z.; Markhasin, E.; Can, T. V.; Corzilius, B.; Tan, K. O.; Barnes, A. B.; Daviso, E.; Su, Y.; Herzfeld, J.; Griffin, R. G. Peptide and protein dynamics and low-temperature/DNP magic angle spinning NMR. J. Phys. Chem. B 2017, 121, 4997-5006.

(32) Lipari, G.; Szabo, A. Effect of vibrational motion on fluorescence depolarisation and nuclear magnetic resonance relaxation in macromolecules and membranes. Biophys. J. 1980, 30, 489-506.

(33) Bloembergen, N. On the interaction of nuclear spins in a crystalline lattice. Physica 1949, 15, 386-426.

(34) Giraud, N.; Blackledge, M.; Böckmann, A.; Emsley, L. The influence of nitrogen-15 proton-driven spin diffusion on the measurement of nitrogen-15 longitudinal relaxation times. J. Magn. Reson. 2007, 184, 51-61.

(35) Mollica, L.; Baias, M.; Lewandowski, J. R.; Wylie, B. J.; Sperling, L. J.; Rienstra, C. M.; Emsley, L.; Blackledge, M. Atomic-resolution structural dynamics in crystalline proteins from NMR and molecular simulation. J. Phys. Chem. Lett. 2012, 3, 3657-3662. 